\title{
Conduction Channels of One-Atom Zinc Contacts
}

\author{
M. Häfner, ${ }^{1}$ P. Konrad, ${ }^{2}$ F. Pauly, ${ }^{1}$ J.C. Cuevas, ${ }^{1}$ and E. Scheer ${ }^{2}$ \\ ${ }^{1}$ Institut für Theoretische Festkörperphysik, Universität Karlsruhe, 76128 Karlsruhe, Germany \\ ${ }^{2}$ Fachbereich Physik, Universität Konstanz, 78457 Konstanz, Germany
}

(Dated: February 2, 2008)

\begin{abstract}
We have determined the transmission coefficients of atomic-sized Zn contacts using a new type of breakjunction which contains a whisker as a central bridge. We find that in the last conductance plateau the transport is unexpectedly dominated by a well-transmitting single conduction channel. We explain the experimental findings with the help of a tight-binding model which shows that in an one-atom $\mathrm{Zn}$ contact the current proceeds through the $4 s$ and $4 p$ orbitals of the central atom.
\end{abstract}

PACS numbers: 73.40.Jn, 73.63.Rt, 74.50.+r

The appearance of experimental techniques such as the scanning tunneling microscope and breakjunctions has allowed to explore the electronic transport at the atomic scale 1 . With these techniques it is possible to gently break a metallic contact and thus form conducting nanowires. During the last stages of the pulling a neckshaped wire connects the two electrodes, the diameter of which is reduced to a single atom upon further stretching. The conductance of these contacts can be described by the Landauer formula: $G=G_{0} \sum_{i} \tau_{i}$, where the sum runs over all the available conduction channels, $\tau_{i}$ is the transmission for the $i^{\text {th }}$ channel and $G_{0}=2 e^{2} / h$ is the quantum of conductance. As it was shown in Ref. 2], the set of transmission coefficients is amenable to measurement in the case of superconducting materials. Using this possibility it has been established that the number of channels in an one-atom contact is determined by the valence of the metal, and the transmission of each channel is fixed by the local atomic environment ${ }^{3,4,5}$. Thus for instance, an one-contact of a monovalent metal such as $\mathrm{Au}$ sustains a single channel, while for $s p$-like metals such as $\mathrm{Al}$ or $\mathrm{Pb}$ one finds three channels due to the contribution of the $p$ orbitals, and in a transition metal such as $\mathrm{Nb}$ the contribution of the $d$ orbitals leads to five channels. Up to now, these attractive ideas have only been tested in four materials ( $\mathrm{Au}, \mathrm{Al}, \mathrm{Pb}$, and $\mathrm{Nb}$ ) due to the need of superconductivity for the channel analysis. In this sense, it would be highly desirable to investigate other groups of metals. An interesting possibility is the analysis of the divalent metals of the IIB group of the periodic table such as $\mathrm{Zn}$. The electronic structure of a $\mathrm{Zn}$ atom is $[\mathrm{Ar}] 3 d^{10} 4 s^{2}$, i.e. the outermost $s$ orbital is full with two electrons. As a solid, $\mathrm{Zn}$ is a conductor due to the overlap between the $4 s$ and the $4 p$ bands. Therefore, for one-atom contacts one expects $\mathrm{Zn}$ to be an intermediate case between the noble metals and Al. The goal of this work is to elucidate what determines the conduction channels of one-atom Zn contacts.

Traditionally, oxidation problems and the gap anisotropy ${ }^{6}$ have prevented the formation of reproducible superconducting contacts with $\mathrm{Zn}$. In order to circumvent these problems we have developed a variation of the mechanically controllable breakjunction (MCB) technique which comprises a Zn whisker as central part. This new technique allows us to fabricate reproducibly oneatom Zn contacts with well-characterized lattice properties of the electrodes. Using the superconducting current-voltage $(I V)$ characteristics ${ }^{2}$ we have reliably extracted the transmission coefficients. We find that in the last plateau, where the conductance is around $0.8 G_{0}$ (Refs. 7 8]), the transport is largely dominated by a single channel. In order to understand these findings we have performed tight-binding calculations of the conductance of one-atom $\mathrm{Zn}$ contacts. Our theoretical results show that the transport takes place through the $4 s$ and $4 p$ bands of $\mathrm{Zn}$. In agreement with the experiment, we obtain for the one-atom case a conductance between 0.8 and $1.0 G_{0}$, and it is dominated by a single channel which is a combination of the $s$ orbital and the $p$ orbital along the transport direction of the central atom.

Our first attempts to investigate $\mathrm{Zn}$ contacts were carried out with both "notched-wire" breakjunctions 9 and thin-film breakjunctions ${ }^{10}$. However, the granu-

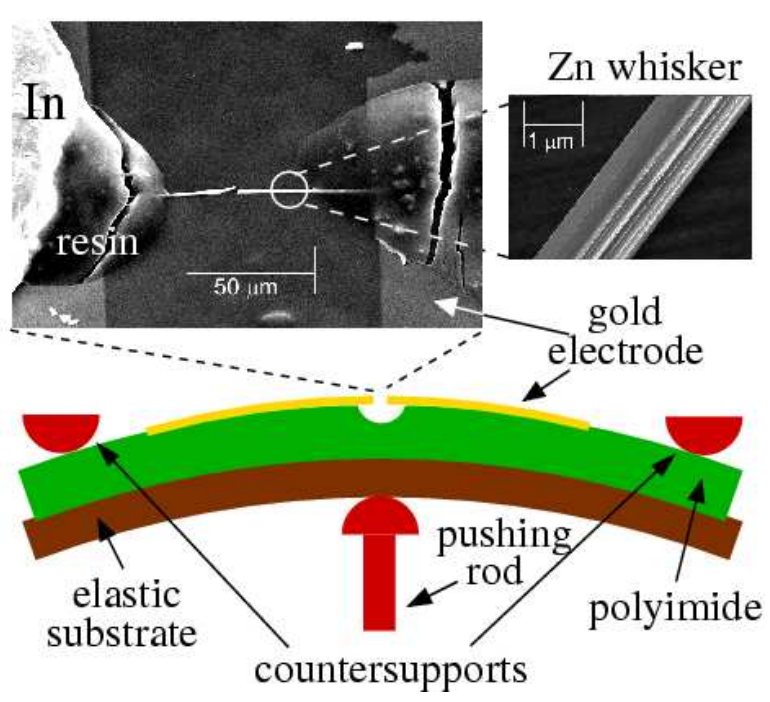

FIG. 1: (Color online) Three-point bending mechanism. The pushing rod bends the bronze substrate. The optical micrograph shows a whisker MCB after measurement. Right inset: blow up of a typical $\mathrm{Zn}$ whisker with a diameter of $1.7 \mu \mathrm{m}$. 


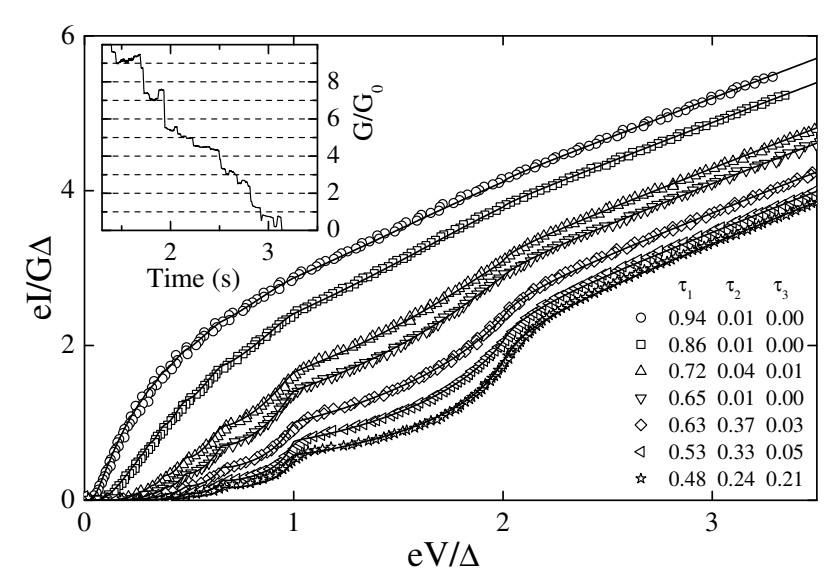

FIG. 2: Current-voltage characteristics of several one-atom contacts of $\mathrm{Zn}$ arranged with a whisker MCB at $T=0.27 \mathrm{~K}$ (symbols) and best fits to MAR theory ${ }^{13.14}$. The transmission coefficients obtained from the fit are indicated in the figure. Inset: typical opening curve recorded at $T=1.5 \mathrm{~K}$.

lar structure of the evaporated films and the intrinsic gap anisotropy of $\mathrm{Zn}$ prevented the observation of reproducible superconducting $I V$ s, which are necessary to obtain the channel transmissions. To solve these problems we have prepared MCBs consisting of $\mathrm{Zn}$ whiskers glued on top of a pre-patterned flexible substrate (see Fig. 1). Whiskers are thin single crystalline needles, which in the case of $\mathrm{Zn}$ grow with a probability of $67 \%$ along the $a$-axis by the so-called pressure method 11 . Following Ref. 11] we have produced whiskers by electroplating 8 to $10 \mu \mathrm{m}$ thick layers of $99.99 \%$ pure Zn onto stainless steel substrates 12 . We apply a uniaxial pressure of $50 \mathrm{~N} / \mathrm{mm}^{2}$ onto a package of 15 substrates, polish its edges carefully and store it at $80{ }^{\circ} \mathrm{C}$ and 200 mbar helium atmosphere. The whiskers start to grow from the edges of the package. The growth saturates after a period of 3 to 6 months, giving rise to whiskers of diameter ranging from 0.5 to 2 $\mu \mathrm{m}$ and length of the order of 0.5 to $1 \mathrm{~mm}$ (see inset of Fig. (1). To contact the whiskers we prepare bronze substrates of size $3 \times 18 \mathrm{~mm}^{2}$ covered with an insulating layer of polyimide and $70 \mathrm{~nm}$ thick gold electrodes separated by $100 \mu \mathrm{m}$. Then, individual whiskers are deposited onto the substrate and mechanically contacted by two small dots of epoxy resin. The electrical contact between the whiskers and the electrodes is obtained by gluing two small pieces of In onto the whiskers and the gold pads. With this method we obtain whisker breakjunctions with resistances of 10 to $100 \Omega$ at room temperature. Finally, we mount them onto a three point bending mechanism ${ }^{9}$ (see Fig. 11) thermally anchored to the base temperature pot $(\approx 260 \mathrm{mK})$ of a ${ }^{3} \mathrm{He}$ cryostat.

Breaking the contact, the conductance evolves as usual in a step-like manner. An example of an opening curve is shown in the inset of Fig. 22 where one sees the typical behavior for $\mathrm{Zn}$ atomic-sized contacts with a conductance

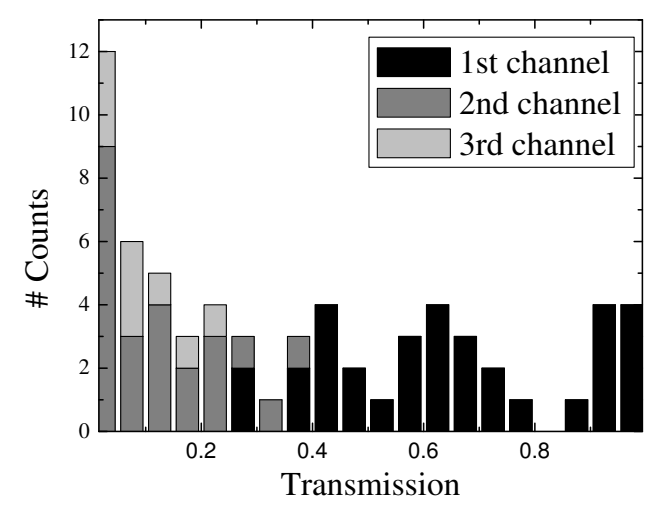

FIG. 3: Experimental histogram of the channel transmissions $\tau_{i}$ obtained in the last conductance plateau of a $\mathrm{Zn}$-whisker MCB. We count all those contacts as belonging to the last plateau that have been recorded when the conductance once dropped to or below $G_{0}$. The channels are labeled as 1st, 2 nd, ... according to their $\tau_{i}^{\prime} s$.

in the last plateau of the order of $0.8 G_{0}$. The stability of the setup allows us to halt at any point along the breaking to record the $I V \mathrm{~s}$. In particular, to determine the superconducting gap the whisker of Fig. [ 1 was broken to form a tunnel contact. In this limit a well-pronounced gap is visible in the $I V \mathrm{~s}$, and a value of $\Delta=160 \mu \mathrm{eV}$ was deduced. This value is close to the expected value for the $x$-direction in real space, corresponding to the crystallographic [110] direction, the direction with the largest gap. Although this suggests that the whisker was grown in this direction, we cannot exclude that this rather high value is due to the proximity effect with the In contacts $\left(2 \Delta_{\text {In }} \simeq 1.05 \mathrm{meV}\right)$. In Fig. 2 we show the superconducting $I V$ s obtained at $0.27 \mathrm{~K}$ for seven different contacts. Together with the data points we also show the best fit obtained with the multiple Andreev reflection (MAR) theory 13,14 at zero temperature. The details of the fit procedure are explained in Ref. [2]. The excellent agreement with the MAR theory allows us to determine the whole set of transmission coefficients $\left\{\tau_{i}\right\}$ with a precision of a few percent (depending on the value of $\tau_{i}$ ) for up to 4 channels. To investigate the conduction channels of the smallest contacts (presumably one-atom contacts), we have collected the transmission coefficients obtained in the last plateau of 33 contact realizations. The results are shown in the histogram of Fig. 3, From this plot we draw two important conclusions: (i) the conductance in the last plateau is largely dominated by a single channel with a high transparency, and (ii) depending on the contact geometry a second and third channels with low transmissions can also contribute to the transport.

In order to understand the experimental findings we have performed conductance calculations following the method described in Refs. 3,5. Briefly, in this approach the electronic structure of the atomic contacts is de- 
scribed in terms of a tight-binding Hamiltonian written in an orthogonal basis. The different on-site energies and hopping elements are taken from the bulk parametrization of Ref. [15], which accurately reproduces the band structure of bulk materials. Our basis is formed by 9 atomic orbitals: $3 d, 4 s, 4 p$, which give rise to the main bands around the Fermi energy, and hopping elements up to third neighbors are taken into account. We have imposed local charge neutrality in all the atoms of the constriction by means of a self-consistent variation of the on-site energies. The leads are described with bulk atoms. The transmission of the contacts is calculated using Green function techniques, which allow us to express the set of transmission coefficients $\left\{\tau_{i}\right\}$ in terms of the microscopic parameters of the atomic contacts.

It is instructive to first discuss the bulk density of states (DOS) of $\mathrm{Zn}$ in its hcp structure, which is shown in Fig. 目(a). Notice that the $d$ band is rather narrow, it lies $\approx 9 \mathrm{eV}$ below the Fermi energy and it is practically full. It is then obvious that the $s$ and $p$ bands will play the main role in the conduction. Since there is an uncertainty in the growth direction of the whiskers, we have studied the conductance of geometries with different crystallographic orientations. In the inset of Fig. 4(b) we show an example of an one-atom contact along the [001] direction (c-axis). The geometry is constructed starting with a single atom and choosing the nearest-neighbors in the next layers. In Fig. 4(b-c) one can see for this geometry the local DOS at the central atom and the transmission of the individual channels $\tau_{i}$ as a function of energy. In the local DOS we see that the $p_{z}$ level $(z$ is the transport direction) is shifted to lower energies due to its better coupling to the leads as compared with $p_{x}$ and $p_{y}$, which remain degenerate in this ideal geometry. This fact implies that the orbital $p_{z}$ plays a more important role in the transport. In the relevant energy range the $d$ band has a very tiny local DOS and it has therefore not been depicted. In Fig. 4(c) we see that at the Fermi energy the total transmission is 0.86 and it is dominated by a single channel with $\tau_{1}=0.81$. The second and third channel are degenerate and their transmission at the Fermi energy is $\tau_{2}=\tau_{3}=0.025$, while the fourth gives a negligible contribution $\tau_{4}=0.001$. To understand the origin of these conduction channels we have analyzed the character of the eigenfunctions of the transmission matrix by looking at their weights in the different orbitals of the central atom. This analysis reveals that the dominant channel is basically a symmetric combination of the $s$ and $p_{z}$ orbitals of the central atom. The second nd third channels are mainly due to the contribution of the $p_{x}$ and $p_{y}$ orbitals. The degeneracy of these two channels is a consequence of the symmetry of this ideal geometry and reflects the degeneracy of the local DOS, see Fig. प (b). Their transmission is rather low because the transport takes place through the tails of the $p_{x}$ and $p_{y}$ bands. The antisymmetric combination of $s$ and $p_{z}$ forms a channel of negligible transmission due to the fact that this combination is orthogonal to the states of the

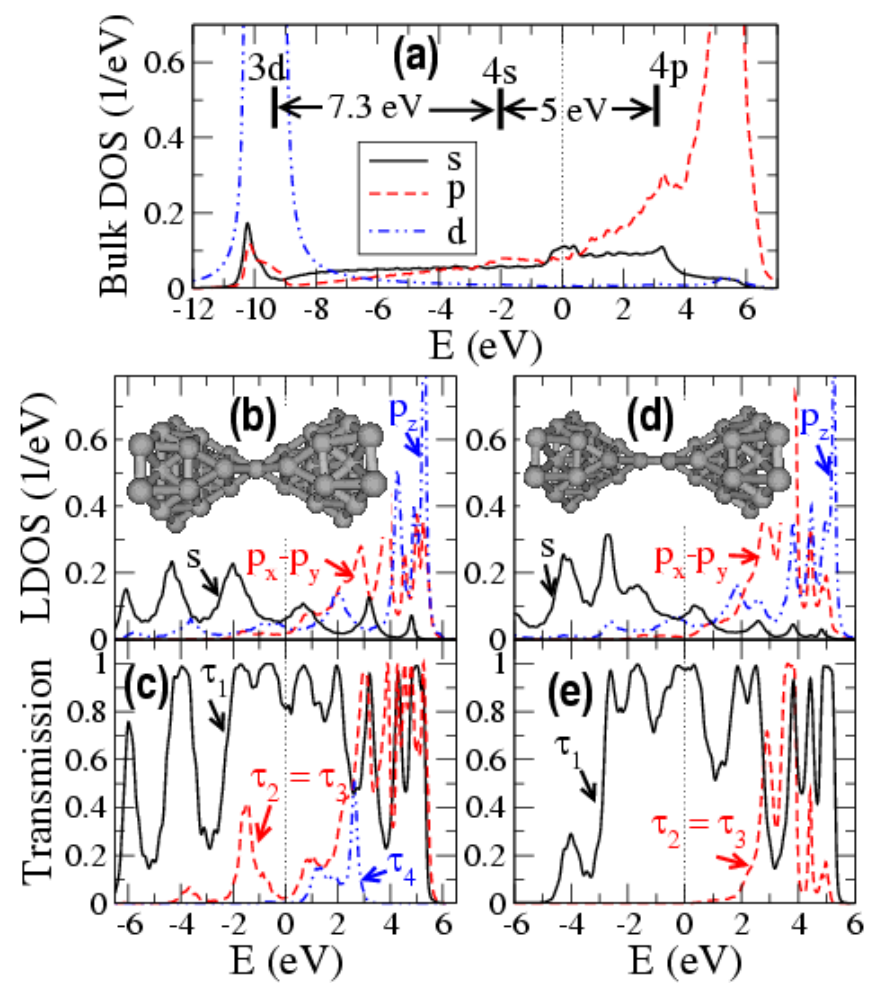

FIG. 4: (Color online) (a) Bulk density of states of Zn hcp projected onto the $s, p$ and $d$ orbitals as a function of energy. The labels indicate the value of the on-site energies. Inset of panel (b): contact along the [001] direction, with a single central atom and containing 45 atoms. The distance between the different atoms is the bulk distance. (b-c) Local DOS at the central atom projected onto the $s$ and $p$ orbitals and channel transmissions as a function of energy for the contact of the inset of panel (b). Notice that the channel $p_{x}-p_{y}$ is two-fold degenerate. Inset of panel (d): the same contact as in panel (b), but with a dimer in the narrowest part. The distance between the central atoms is the bulk nearest-neighbor distance. (d-e) The same as in panels (b-c) for the dimer geometry. The Fermi energy is set to zero and indicated with vertical lines.

leads. So in short, the nature of the channels in this oneatom $\mathrm{Zn}$ contact is similar to the $\mathrm{Al}$ case $^{3}$. The main difference is that $\mathrm{Zn}$ has one valence electron less than Al. Thus, the Fermi energy is lower and consequently lies further away from the center of the $p$ bands, resulting in a lower transmission of the $p_{x}-p_{y}$ channels.

As explained above, the second and third channels have a low transmission due to the fact that the transport takes place through the tails of the $p_{x}$ and $p_{y}$ bands. This suggests that a reduction of the coupling to these orbitals could result in a negligible contribution of these channels. To test this idea we have analyzed one-atom contacts with a dimer in the narrowest part of the contact, see inset of Fig. 4(d). This type of geometry has been frequently observed in molecular dynamics simulations of atomic contacts of $\mathrm{Al}$ (Ref. [16]) and $\mathrm{Au}$ (Ref. [1] ). In Fig. [4(d-e) the local DOS in one of the central atoms and 

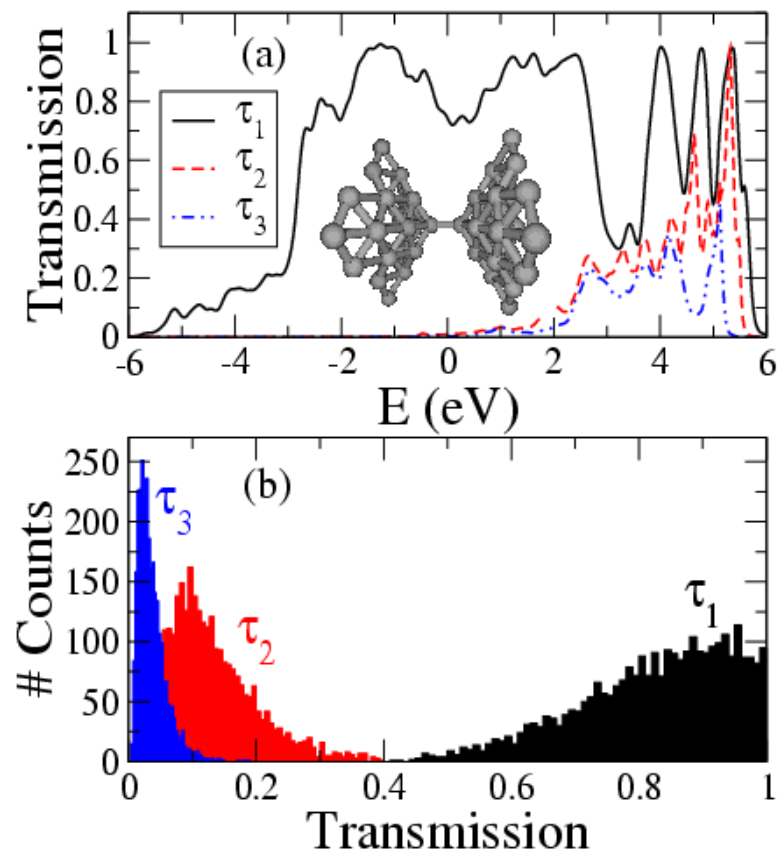

FIG. 5: (Color online) (a) Channel transmissions as a function of energy for the contact of the inset, which is grown along the [110] direction, has a dimer in the middle and contains 60 atoms. The Fermi energy is set to zero. (b) Histograms of the transmission coefficients for 3000 disorder realizations (see text) of the one-atom contact of the inset of Fig. 4(b).

the transmission through this dimer contact are shown. The total transmission at the Fermi energy is 0.97, and as suspected, it is completely dominated by a single channel $\left(\tau_{2}=4 \times 10^{-4}\right)$, while the character of the channels is the same as in the case analyzed above. Thus, by changing the contact geometry from a short contact with a single atom in the constriction to a long contact in a dimer configuration the conductance may increase by about 0.1 $G_{0}$. Opening traces that support this prediction are reported in the literature ${ }^{7.8}$ and have also been found in the present experiment (see inset of Fig 2).

Let us now discuss the results for contacts along the [110] direction (a-axis). An example of a contact geometry with a dimer configuration and its corresponding transmission is shown in Fig. [5] As in the case of the [001] direction, the total transmission $\left(\tau_{t o t}=0.77\right)$ is clearly dominated by a single channel $\left(\tau_{1}=0.75\right)$, which is formed by a symmetric combination of the $s$ and $p_{x}$ orbitals of the central atoms ( $x$ is now the transport direction). The second channel is on the border of what is measurable $\left(\tau_{2}=0.01\right)$, while the transmission of the third one is $\tau_{3}=0.004$. Notice that in this case these two channels are not degenerate. We find that the transmission for contacts along the [110] direction is slightly lower than for the [001] one, which we attribute to the larger apex angle in the first direction, which produces less adiabatic contacts. These findings are in agreement with the observation of Ref. [8] where a splitting of the first peak of the histogram into two sub-peaks with 0.7 and $0.9 G_{0}$ was reported.

For all the geometries analyzed in this work we have checked that the results do not change qualitatively with the number of atoms in the constriction region. We have also studied the influence of disorder, which we simulate by changing randomly the positions of the atoms in the contact region with a very large maximum amplitude of $20 \%$ of the nearest-neighbor distance. The hoppings are then computed using the scaling laws proposed in Ref. [15]. In Fig. 5(b) we show a histogram of the individual transmissions for 3000 realizations of contacts along the [001] direction with a single central atom. As it can be seen, the transmission is still dominated by a single channel, and depending on the local environment of the central atom a second and even a third channel can have a measurable contribution, in agreement with the experimental results (see Fig. 3). Similar histograms for the dimer geometries show that $\tau_{2}$ and $\tau_{3}$ typically lie below the measurement threshold.

In summary, we have presented an experimental and theoretical study of the conduction channels in $\mathrm{Zn}$ atomic junctions. We have shown that, although $\mathrm{Zn}$ is a divalent metal, the conductance of one-atom contacts is dominated by a single well-transmitting channel, which we have traced back to the symmetry of the valence orbitals $(s$ and $p$ ) of the central atom. Our results constitute a new illustration of how the electronic structure of an atom determines the conductance of the circuit in which it is embedded.

This work has been financed by the SFB 195, SFB 513, the Alfried Krupp von Bohlen und Halbach-Stiftung, the Landesstiftung Baden-Württemberg and the Helmholtz Gemeinschaft (contract VH-NG-029).
1 For a recent review see N. Agraï, A. Levy Yeyati, and J.M. van Ruitenbeek, Phys. Rep. 377, 81 (2003).

2 E. Scheer, P. Joyez, D. Esteve, C. Urbina, and M.H. Devoret, Phys. Rev. Lett. 78, 3535 (1997).

3 J.C. Cuevas, A. Levy Yeyati, and A. Martín-Rodero, Phys. Rev. Lett. 80, 1066 (1998).

4 E. Scheer, N. Agraït, J.C. Cuevas, A. Levy Yeyati, B. Ludoph, A. Martín-Rodero, G. Rubio, J.M. van Ruitenbeek, and C. Urbina, Nature 394, 154 (1998).
5 J.C. Cuevas, A. Levy Yeyati, A. Martín-Rodero, G. Rubio, C. Untiedt, and N. Agraït, Phys. Rev. Lett. 81, 2990 (1998).

${ }^{6}$ C.R. Cleavelin and B.J. Marshall, Phys. Rev. B 10, 1902 (1974).

7 A.I. Yanson, Ph.D thesis, University of Leiden, The Netherlands (2001).

8 A. Mayer-Gindner, H. v. Löhneysen, and E. Scheer, submitted 
9 C.J. Muller, J.M. van Ruitenbeek, and L.J. de Jong, Physica (Amsterdam) 191C, 485 (1992).

10 J.M. van Ruitenbeek, A. Alvarez, I. Piñeyro, C. Grahmann, P. Joyez, M.H. Devoret, D. Esteve, and C. Urbina, Rev. Sci. Instrum. 67, 108 (1996).

${ }^{11}$ U. Schulz, P.J. Wilbrandt, and R. Tidecks, J. Crystal Growth 85, 472 (1987).

12 A detailed description of the fabrication procedure will be published elsewhere.

13 D. Averin and A. Bardas, Phys. Rev. Lett. 75, 1831 (1995).
14 J.C. Cuevas, A. Martín-Rodero, A. Levy Yeyati, Phys. Rev. B 54, 7366 (1996).

15 D.A. Papaconstantopoulos, Handbook of the band structure of elemental solids, Plenum Press, New York (1986).

16 P. Jelínek, R. Pérez, J. Ortega, and F. Flores, Phys. Rev. B 68, 085403 (2003).

17 M. Dreher, J. Heurich, J.C. Cuevas, E. Scheer, and P. Nielaba, cond-mat/0406281 